\title{
A model integrating Killer Immunoglobulin-like Receptor (KIR) haplotypes for risk prediction of COVID-19 clinical disease severity
}

\author{
Meral Beksac ${ }^{1}$ (1) Hasan Yalim Akin ${ }^{1} \cdot$ Emine Begum Gencer-Oncul ${ }^{1} \cdot$ Mahsa Yousefzadeh $^{1} \cdot$ Guldane Cengiz Seval $^{1}$. \\ Ezgi Gulten ${ }^{2} \cdot$ Irem Akdemir Kalkan ${ }^{2} \cdot$ Gule Cinar $^{2} \cdot$ Osman Memikoglu $^{2} \cdot$ Ergun Karaagaoglu $^{3} \cdot$ Klara Dalva $^{4}$
}

Received: 24 May 2021 / Accepted: 14 August 2021 / Published online: 18 September 2021

(c) The Author(s), under exclusive licence to Springer-Verlag GmbH Germany, part of Springer Nature 2021

\begin{abstract}
Associations between inherited Killer Immunoglobulin-like Receptor (KIR) genotypes and the severity of multiple RNA virus infections have been reported. This prospective study was initiated to investigate if such an association exists for COVID-19. In this cohort study performed at Ankara University, 132 COVID-19 patients (56 asymptomatic, 51 mild-intermediate, and 25 patients with severe disease) were genotyped for KIR and ligands. Ankara University Donor Registry (n:449) KIR data was used for comparison. Clinical parameters (age, gender, comorbidities, blood group antigens, inflammation biomarkers) and KIR genotypes across cohorts of asymptomatic, mild-intermediate, or severe disease were compared to construct a risk prediction model based on multivariate binary logistic regression analysis with backward elimination method. Age, blood group, number of comorbidities, CRP, D-dimer, and telomeric and centromeric KIR genotypes (tAA, tAB1, and cAB1) along with their cognate ligands were found to differ between cohorts. Two prediction models were constructed; both included age, number of comorbidities, and blood group. Inclusion of the KIR genotypes in the second prediction model exp $(-3.52+1.56$ age group - 2.74 blood group (type A vs others $)+1.26$ number of comorbidities -2.46 tAB1 with ligand +3.17 tAA with ligand) increased the predictive performance with a $92.9 \%$ correct classification for asymptomatic and $76 \%$ for severe cases (AUC: $0.93 ; P<0.0001,95 \%$ CI 0.88, 0.99). This novel risk model, consisting of KIR genotypes with their cognate ligands, and clinical parameters but excluding earlier published inflammation-related biomarkers allow for the prediction of the severity of COVID-19 infection prior to the onset of infection. This study is listed in the National COVID-19 clinical research studies database.
\end{abstract}

Keywords SARS-CoV-2 · COVID-19 · Killer Immunoglobulin-like Receptor (KIR) · Natural killer (NK) cells

\section{Introduction}

Despite intense scientific research and major achievements in vaccines, SARS-CoV-2 is still a major cause of morbidity and mortality worldwide. The current question as to why some

Ergun Karaagaoglu and Klara Dalva contributed equally.

Meral Beksac

beksac@medicine.ankara.edu.tr

1 Department of Hematology, Faculty of Medicine, Ankara University, Ankara, Turkey

2 Department of Infectious Diseases and Clinical Microbiology, Faculty of Medicine, Ankara University, Ankara, Turkey

3 Department of Biostatistics, Faculty of Medicine, Hacettepe University, Ankara, Turkey

4 Department of Hematology, Immunogenetics Laboratory, Faculty of Medicine, Ankara University, Ankara, Turkey individuals develop an asymptomatic infection while others become critically ill has not been completely answered (Vetter et al. 2020). Viral load, early intervention, gender, blood groups, comorbidities, and inherited genotypic variants may be significant variables (Vetter et al. 2020; Wu et al. 2021; van der Made et al. 2020). Several groups have tried to develop risk scores based on clinical features, either alone or combined with acute phase reactants (Hu et al. 2020; Zhou et al. 2020). COVID-19 Genetics Research groups have examined several immune response-related variants already identified as conferring susceptibility to other viral infections (Ovsyannikova et al. 2020; Casanova et al. 2020). An early study conducted in Spain and Italy identified a gene cluster on chromosome 3 as a genetic susceptibility locus for COVID-19-associated respiratory failure (Severe Covid-19 GWAS Group et al. 2020). These results were later confirmed by the COVID-19 Host Genetics Initiative and the Genetics of Mortality in Critical Care (GenOMICC) GWAS (Pairo-Castineira et al. 2021; The COVID-19 Host Genetics Initiative 2020). Chromosome 3 
locates a risk locus harboring immune genes such as $C C R 9$, CXCR6, XCR1, CCR1, and CCR2, inherited from Neanderthals and carried by around $50 \%$ of people in South Asia and $16 \%$ of people in Europe (Zeberg and Paabo 2021; Zeberg and Paabo 2020). From the GenOMICC study, additional genetic variants in IFNAR2 and OAS2 were proposed to be associated with a critical illness (Pairo-Castineira et al. 2021; Zhou et al. 2021). The COVID Human Genetic Effect Consortium reported an overall estimated 3.5\% incidence of TLR3, IRF7, and IFNAR1 variants among patients with severe disease suggesting a defective innate immune response. Another approach identified rare putative loss-of-function variants of $\mathrm{X}$-chromosomal $T L R 7$ that were associated with impaired type I and II IFN responses (Made et al. 2020). The same group also identified another Neanderthal gene, a DPP4 variant, observed in $\sim 1 \%$ of Europeans, $2.5 \%$ of South Asians, $\sim 4 \%$ of East Asians, $\sim 0.7 \%$ of admixed Americans, and absent among Africans south of the Sahara, which doubles the risk of becoming critically ill (Zeberg 2020). These findings strongly point toward a causal link between inherited variants and the severity of COVID-19 infection.

Natural killer (NK) cells play a critical role in immune regulation in autoimmunity, infections, and cancer (Beksac et al. 2015; Sahin et al. 2018). The NK cell functions are determined by a broad array of activating and inhibitory receptors. There is increasing evidence suggesting a role for KIR genotypes in anti-viral immunity as well as reports on KIR disease associations (Khakoo and Carrington 2006). A more recent approach based on analysis of the genomic organization of individual genes allows for the identification of KIR haplotypes (Vendelbosch et al. 2015; Roe et al. 2017; Cisneros et al. 2020). Haplotypic analysis of KIR diversity allows for the inclusive evaluation of the simultaneous effects of both inhibitory and activating KIR genotypes on immune responsiveness.

Although there are groups that have reported associations between viral infections and KIR gene and/or ligand frequencies as well as with A/B KIR haplotypes, to our knowledge, there are none that have focused on telomeric/centromeric KIR motifs. Lu et al. (Lu et al. 2008) observed lower frequencies of haplotype $\mathrm{A}$ and higher frequencies of haplotype B in HBV-infected patients compared to the healthy controls. They have also found an association with certain KIR genotypes in Caucasian populations. However, they did not include telomeric/centromeric motifs in their study (Lu et al. 2008). In the context of RNA virus infections, Influenza A (H1N1), HIV, HCV, Ebola, Dengue virus (DENV), and Chikungunya virus (CHIKV) have been associated with specific KIR genes/ligands. KIR/HLA compound genotypes with an activating profile (presence of activating KIR or lack of inhibitory KIRs in the presence of their cognate ligands) are associated with resistance to HCV (Khakoo et al. 2004) and HIV infections (Jennes et al. 2006), and slower HIV disease progression (Martin et al. 2002). On the other hand, Shan et al. (2018) found that chronic HCV infection was associated with KIR2DL3/2DL3, 2DL3/2DL3 + HLA-C1, or C1C1 genotypes. Similarly, co-existence of KIR2DL3, which is included in the centromeric AA (cAA) motif and the cognate ligand $\mathrm{C} 1$ or $\mathrm{C} 2$, was linked to rapid progression/susceptibility to HIV and Chikungunya virus (CHIKV) infection, respectively (Mori et al. 2019). Ahlenstiel et al. (2008) and La et al. (2011) and have demonstrated a possible association with NK cell dysfunction in patients with overactive immune responses to $\mathrm{H} 1 \mathrm{~N} 1 / 09$, leading to severe disease. Individuals with KIR3DL1/S1 lacking Bw4 or KIR2DL1 lacking HLA$\mathrm{C} 2$ ligands were overrepresented in ICU patients during the 2009 flu pandemic, as were individuals with KIR2DL2/L3 and its cognate ligand HLA-C1. Aranda-Romo et al. (2012) found an association between severe H1N1 infections and KIR2DL5, KIR2DS5, and KIR3DS1 genes. Although these authors did not analyze KIR haplotypes, these data suggest that $\mathrm{tAB} 1$ without its ligand or centromeric $\mathrm{AB} 1$ (cAB1) with its ligand are associated with ICU admission during H1N1 infection (Ahlenstiel et al. (2008); La et al. (2011). However, tAB1 with its ligand is protective against dengue fever (Chaisri et al. 2020). Likewise, the protective genotypes suggested by Jiang et al. (2013) and Shan et al. (2018) may correlate with $\mathrm{tAB} 1$ and $\mathrm{cAA}$ motifs along with their ligands and may therefore be associated with the reduced severity of HIV and HCV infections, respectively. Also, the association found between KIR2DS3-C1 combination and HIV, HCV, and CHIKV infections may be mediated through the cAA genotype (Shan et al. 2018; Mori et al. 2019; Petitdemange et al. 2014).

To date, there have been only two published reports on the frequency of KIR receptors and their ligands in relation to the severity of COVID-19. The first study from National Research Project for SARS, Beijing Group reported a reduced number of CD158b (KIR2DL3, a member of the centromeric A haplotype) expressing NK cells among severely affected patients compared to mild cases in accordance with earlier findings reported for influenza (Ahlenstiel et al. 2008; Soleimanian and Yaghobi 2020). In the second study, an extensive phenotypic and functional analysis of KIR genotypes and KIR receptor expression was performed by Maucourant et al. (2020) who did not report on KIR genotype or haplotype associations with disease severity. Thus, our current study is the first that attempts to find an association between KIR genotypes and COVID-19.

In this prospective study, we aimed to analyze and compare the frequencies of KIR haplotype or haplotype regions in patients who were diagnosed with COVID-19 and who were classified as either asymptomatic, mild-intermediate, or severe in order to develop a prediction model for disease severity. 


\section{Results}

The clinical parameters, number of comorbidities, and age group classifications are summarized in Table 1. During the study period of five months, a total of 132 patients were included. One hundred four of these were recruited prospectively (original cohort). We were able to acquire 28 additional COVID-19 diagnosed subjects from Ankara University Donor Registry retrospectively. The age distribution of both cohorts of patients was similar (original cohort: median 44 (18-89) vs registry patients: median 43 (25-76). Patients recruited from the Registry cohort were either asymptomatic ( $n: 4)$ of which only one had one comorbidity or had presented with mild-intermediate symptoms (one: 4; two: 6; three: one comorbidity) with a total comorbidity frequency of $43 \%$. Among the original cohort, the frequency of comorbidities (total $45 \%$ with one $17 \%$, two $12 \%$, three $6 \%$ ) was similar to the patients recruited from the KIR genotyped Registry. All but eight patients recovered, resulting in $18.93 \%$ severe cases and a mortality rate of $6 \%$. Comparisons between asymptomatic, mild-intermediate, and severe cases showed a positive correlation with age, comorbidities, C-reactive protein (CRP), and D-dimer values (Table 1). Blood groups differed across all comparisons $(P=0.10)$. Figure 1 illustrates the description of KIR genotypes based on the chromosomal location of KIR genes. For KIR genotype comparisons, the most frequent motifs in normal populations were chosen: telomeric tAA, tB1B1, tB2B2, tAB1, tAB2, tB1B2 and centromeric cAA, cB1B1, cB2B2, cAB1, cAB2, cB1B2. This analysis showed tAA and $\mathrm{CAB} 1$ incidence (with or without ligand dependence) to be higher among cases with severe disease (Table 1). On the contrary, tAB1 genotype frequency, with or without the corresponding ligands, was more frequently found in asymptomatic patients (Table 1). KIR2DS5 which is expressed on the cell surface, unlike KIR2DS3, showed a decreasing frequency from asymptomatic patients (57.1\%) to mild-intermediate (47.1\%) to those with severe infection (28\%). As KIR genotypes tAB1 and tAB2 differ by KIR2DS5 vs KIR2DS3, we merged them and tested tAB instead of $\mathrm{ABB} 1$ or $\mathrm{ABB} 2$; no significant associations were seen. All subgroup analyses performed in order to reduce ambiguity did not change the results.

Among asymptomatic patients, $19.64 \%$ had CRP $>10 \mathrm{mg} / \mathrm{L}$. When we compared the $\mathrm{AB} 1$ genotype frequency between healthy controls, asymptomatic cases, and high-CRP asymptomatic patients, we observed a gradual increase from $29.13 \%$ $(17.48 \% \mathrm{w}$ ligand) to $50 \%$ (30.36\% w ligand) to $54.55 \%$ (36.36 w ligand), suggesting a protective role for tAB1. A similar analysis for tAA carrying only one activating KIR showed $50.97 \%$ (17.73\% w ligand), $28.57 \%$ (3.57\% w ligand), and $18.18 \%$ (zero w ligand), respectively, thereby suggesting an inverse association with CRP response.
Multivariate Binary Logistic Regression with Backward Elimination Method was used to identify the significant factors that could be used to discriminate between the "asymptomatic" and the "severe disease" groups. The first model consisted of age, comorbidity, gender, and blood group, and telomeric and centromeric genotypes (tAA, tAB1, and $\mathrm{cAB} 1$ ) were added to the second model. The performances of our prediction models for COVID19-related severe disease risk were evaluated by constructing two ROC curves, one with and the other without KIR genotypes; they are shown in Fig. 2. Since CRP and D-dimer were not measured in all patients, the inclusion of these two parameters would cause a decrease in the sample size; they were therefore excluded from the multivariate analysis.

Age group, blood group A, number of comorbidities, $\mathrm{ABB} 1$ with ligands $\mathrm{C} 2+\mathrm{Bw} 4$, and tAA with ligands $\mathrm{Bw} 4+\mathrm{ABw} 4$ were found to be the significant independent variables for predicting severe disease. The multivariate logistic model exp(-3.52 +1.56 age group -2.74 blood group A + 1.26 number of comorbidities $-2.46 \mathrm{tAB} 1 \mathrm{w}$ ligand +3.17 tAA w ligand) had an overall correct classification rate of $87.65 \%$ with $92.86 \%$ correct classification for asymptomatic cases and $76 \%$ for severe disease (Table 2). The number of comorbidities was in a range of 0 to 4; age was classified into 4 groups and coded as follows: under 35 years as 1, 35-50 years as 2, 51-64 years as 3, and above 65 years of age as 4 . Blood group type was dichotomized as blood group A vs others. While the AUC for the model without KIR genotypes was $0.87 ; P<0.0001$ (95\% CI 0.79, 0.96 ); AUC for the model including the KIR genotypes was $0.93 ; P<0.0001(95 \%$ CI $0.88,0.99)$ indicating an excellent classification (Fig. 2).

The distribution of KIR genotypes among our COVID19 patients can be compared with the different populations worldwide (Human Genome Diversity Project, HGDP, Hollenbach et al. 2012) and also our normal stem cell donor distributions presented in Fig. 1 and Table 3. Since tAB1 was only recognized after the publication of the HGDP, tAB1 data could only be included for our cases. Still, it is possible to observe similarities between Turkish, European, and Central-South Asian populations with regard to $\mathrm{AAA}$. Regarding $\mathrm{tAB}$ frequencies, similarities exist only between Turkish and European subjects.

\section{Discussion}

To the best of our knowledge, this is the first study to integrate patient characteristics including age, blood group, and comorbidities with KIR motifs in a model for the prediction of severe COVID-19 infection requiring ICU admission. Our model has produced higher odds ratios when compared with earlier attempts. Risk scoring models 
Table 1 Clinical and KIR genotype characteristics of COVID-19 diagnosed patients in comparison with clinical infection severity

\begin{tabular}{|c|c|c|c|c|c|}
\hline $\begin{array}{l}\text { COVID-19 patient characteristics } \\
n(\%)\end{array}$ & Asymptomatic $(n=56)$ & $\begin{array}{l}\text { Mild-intermediate } \\
(n=51)\end{array}$ & $\begin{array}{l}\text { Severe } \\
(n=25)\end{array}$ & $\begin{array}{l}\text { Symptomatic } \\
\text { (mild-intermediate } \\
\text { plus severe) } \\
(n=76)\end{array}$ & $P$ value ${ }^{\mathrm{A}}$ \\
\hline Gender (male/female) & $\begin{array}{l}23 / 33 \\
(41.07 / 58.93 \%)\end{array}$ & $\begin{array}{l}23 / 28 \\
(45.10 / 54.90 \%)\end{array}$ & $\begin{array}{l}15 / 10 \\
(40.00 / 60.00 \%)\end{array}$ & $\begin{array}{l}38 / 38 \\
(50.00 / 50.00 \%)\end{array}$ & $P=0.282$ \\
\hline Age $[$ mean $(\mathrm{SD})]$ & $34.2(12.8)$ & $45.5(15.2)$ & $64.4(18.3)$ & $51.7(18.5)$ & $P<0.0001$ \\
\hline Age $<35$ & $28(50.00 \%)$ & $17(33.33 \%)$ & $3(12.00 \%)$ & $20(26.32 \%)$ & $P<0.0001$ \\
\hline $35 \geq$ age $\leq 50$ & $17(30.36 \%)$ & $14(27.45 \%)$ & $3(12.00 \%)$ & $17(22.37 \%)$ & \\
\hline $51 \geq$ age $\leq 64$ & $9(16.07 \%)$ & $15(29.41 \%)$ & $4(16.00 \%)$ & $19(25.00 \%)$ & \\
\hline Age $\geq 65$ & $2(3.57 \%)$ & $5(9.80 \%)$ & $15(60.00 \%)$ & $20(26.32 \%)$ & \\
\hline Diabetes mellitus & $3(5.36 \%)$ & $6(11.76 \%)$ & $9(36.00 \%)$ & $15(19.74 \%)$ & $P=0.001$ \\
\hline Hypertension & $5(8.93 \%)$ & $15(29.41 \%)$ & $16(64.00 \%)$ & $31(40.79 \%)$ & $P<0.0001$ \\
\hline $\begin{array}{l}\text { Chronic obstructive pulmonary disease } \\
\text { (COPD) }\end{array}$ & $0(0.00 \%)$ & $1(1.96 \%)$ & $5(20.00 \%)$ & $6(7.89 \%)$ & $P<0.001$ \\
\hline Coronary artery disease & $3(5.36 \%)$ & $6(11.76 \%)$ & $2(8.00 \%)$ & $8(10.53 \%)$ & $P=0.499$ \\
\hline Chronic kidney disease (CKD) & $3(5.36 \%)$ & $0(0.00 \%)$ & $3(12.00 \%)$ & $3(3.95 \%)$ & $P=0.046$ \\
\hline Cancer & $0(0.00 \%)$ & $2(3.92 \%)$ & $1(4.00 \%)$ & $3(3.95 \%)$ & $P=0.306$ \\
\hline Obesity & $0(0.00 \%)$ & $0(0.00 \%)$ & $2(8.00 \%)$ & $2(0.03 \%)$ & $P=0.035$ \\
\hline Number of comorbidities $(n=0)$ & $47(83.93 \%)$ & $30(58.82 \%)$ & $7(28.00 \%)$ & $37(48.68 \%)$ & $P<0.0001$ \\
\hline Number of comorbidities $(n=1)$ & $6(10.71 \%)$ & $11(21.57 \%)$ & $6(24.00 \%)$ & $17(22.37 \%)$ & \\
\hline Number of comorbidities $(n=2)$ & $1(1.79 \%)$ & $8(15.69 \%)$ & $7(28.00 \%)$ & $15(19.74 \%)$ & \\
\hline Number of comorbidities $(n=3)$ & $2(3.57 \%)$ & $2(3.92 \%)$ & $4(16.00 \%)$ & $6(7.89 \%)$ & \\
\hline Number of comorbidities $(n=4)$ & $0(0.00 \%)$ & $0(0.00 \%)$ & $1(4.00 \%)$ & $1(1.32 \%)$ & \\
\hline Pulmonary involvement (tomography) & $0(0.00 \%)$ & $7(13.73 \%)$ & $16(64.00 \%)$ & $23(30.26 \%)$ & $P<0.0001$ \\
\hline $\begin{array}{l}\text { C-reactive protein }(\mathrm{mg} / \mathrm{L}) \text { [median }(\min - \\
\max )]\end{array}$ & $4(0.3-281)$ & $12(0.8-159)$ & $178.6(49.4-395.7)$ & $91.9(0.8-395.7)$ & $P<0.0001$ \\
\hline D-Dimer (ng/mL) [median (min-max)] & $141(36-2714)$ & $248(69-2519)$ & $2883(159-46,238)$ & $602(69-46,238)$ & $P<0.001$ \\
\hline Blood group type A & $37(66.07 \%)$ & $23(46.94 \%)$ & $12(48.00 \%)$ & $35(47.30 \%)$ & $P=0.10^{\mathrm{B}}$ \\
\hline Blood group type B & $6(10.71 \%)$ & $11(22.45 \%)$ & $2(8.00 \%)$ & $13(17.57 \%)$ & \\
\hline Blood group type $\mathrm{AB}$ & $5(8.93 \%)$ & $2(4.08 \%)$ & $2(8.00 \%)$ & $4(5.41 \%)$ & \\
\hline Blood group type 0 & $8(14.29 \%)$ & $13(26.53 \%)$ & $9(36.00 \%)$ & $22(29.73 \%)$ & \\
\hline Haplotype AA & $7(12.50 \%)$ & $8(15.69 \%)$ & $6(24.00 \%)$ & $14(18.42 \%)$ & $P=0.425$ \\
\hline Haplotype BB & $8(14.29 \%)$ & $10(19.61 \%)$ & $3(12.00 \%)$ & $13(17.11 \%)$ & $P=0.632$ \\
\hline Haplotype AB & $41(73.21 \%)$ & $33(64.71 \%)$ & $16(64.00 \%)$ & $49(64.47 \%)$ & $P=0.566$ \\
\hline tAA ligand-independent & $16(28.57 \%)$ & $25(49.02 \%)$ & $13(52.00 \%)$ & $38(50.00 \%)$ & $P=0.045$ \\
\hline tAA with ligand ( $\mathrm{Bw} 4+\mathrm{ABw} 4+)$ & $2(3.57 \%)$ & $6(11.76 \%)$ & $5(20.00 \%)$ & $11(14.47 \%)$ & $P=0.061$ \\
\hline tAB1 ligand-independent & $28(50.00 \%)$ & $16(31.37 \%)$ & $5(20.00 \%)$ & $21(27.63 \%)$ & $P=0.020$ \\
\hline tAB1 with ligand $(\mathrm{C} 2+\mathrm{Bw} 4+)$ & $17(30.36 \%)$ & $3(5.88 \%)$ & $4(16.00 \%)$ & $7(9.21 \%)$ & $P=0.004$ \\
\hline tBB ligand-independent & $3(5.36 \%)$ & $6(11.76 \%)$ & $2(8.00 \%)$ & $8(10.53 \%)$ & $P=0.459$ \\
\hline tBB with ligand $(\mathrm{C} 2+\mathrm{Bw} 4+)$ & $1(1.79 \%)$ & $3(5.88 \%)$ & $1(4.99 \%)$ & $4(5.26 \%)$ & $P=0.540$ \\
\hline cAB1 ligand-independent & $15(26.79 \%)$ & $20(39.22 \%)$ & $11(44.00 \%)$ & $31(40.79 \%)$ & $P=0.228$ \\
\hline cAB1 with ligand $(\mathrm{C} 1+\mathrm{C} 2+)$ & $7(12.50 \%)$ & $12(23.53 \%)$ & $9(36.00 \%)$ & $21(27.63 \%)$ & $P=0.050$ \\
\hline
\end{tabular}

${ }^{A}$ Comparison of characteristics among the groups of asymptomatic, mild-intermediate, and severe cases. For categorical variables, Pearson's chi-square or Fisher's exact test were used. For continuous variables, one-way ANOVA for the normally distributed variables and KruskalWallis test for the non-normally distributed variables were used

${ }^{\mathrm{B}}$ Blood type A vs others

based on patient- and disease-related features have been reported; however, almost all of these models include biomarkers which are dynamic, and which develop after the onset of disease (Rod et al. 2020).
Geng et al. (2021) analyzed 12,647 subjects from China and identified male gender, increasing age, the presence of symptoms, delayed diagnosis, comorbidities, and exposure history as variables having an effect on the severity of 


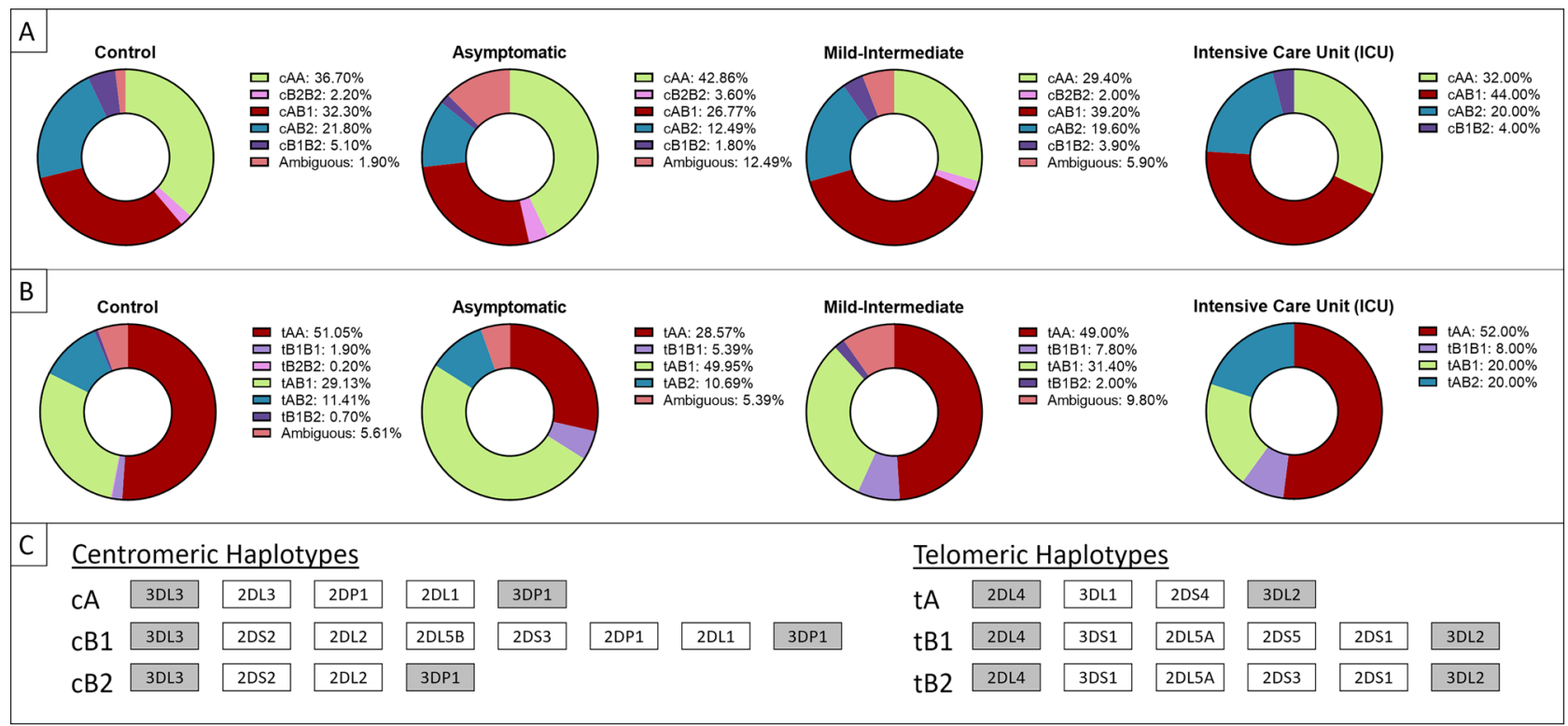

Fig. 1 Distribution of centromeric (A) and telomeric (B) KIR haplotypes, independent of ligands, based on the chromosomal locations (C) among control group and COVID-19 patient groups (grey boxes represent the framework genes)

COVID-19 (OR: 0.53-5.97). In a meta-analysis of twentytwo published reports, the authors found raised CRP, interleukin-6, lactate dehydrogenase (LDH), neutrophil count, \%PD-1 expression, D-dimer, creatinine, AST and cortisol and a low lymphocyte count, a low albumin level, and comorbidities to be linked with illness severity (Khodeir et al. 2021). They reported odds ratios ranging between 2 and 4 based on age and comorbidities. However, risk prediction based on acute phase reactants may not be an optimal approach as the information is highly dynamic and is only obtained after the onset of symptoms.

In a cohort of 1590 patients from China, Liang et al. (2020) constructed a risk calculator to estimate COVID-19 disease severity which included ten predictive factors, all determined at admission into their scoring model, as follows: chest radiographic abnormalities, age, hemoptysis,
Fig. 2 ROC curves show the efficacy of prediction of severe disease for COVID-19 by our model constructed with (the bold line) or without KIR genotypesVisual abstract of this manuscript

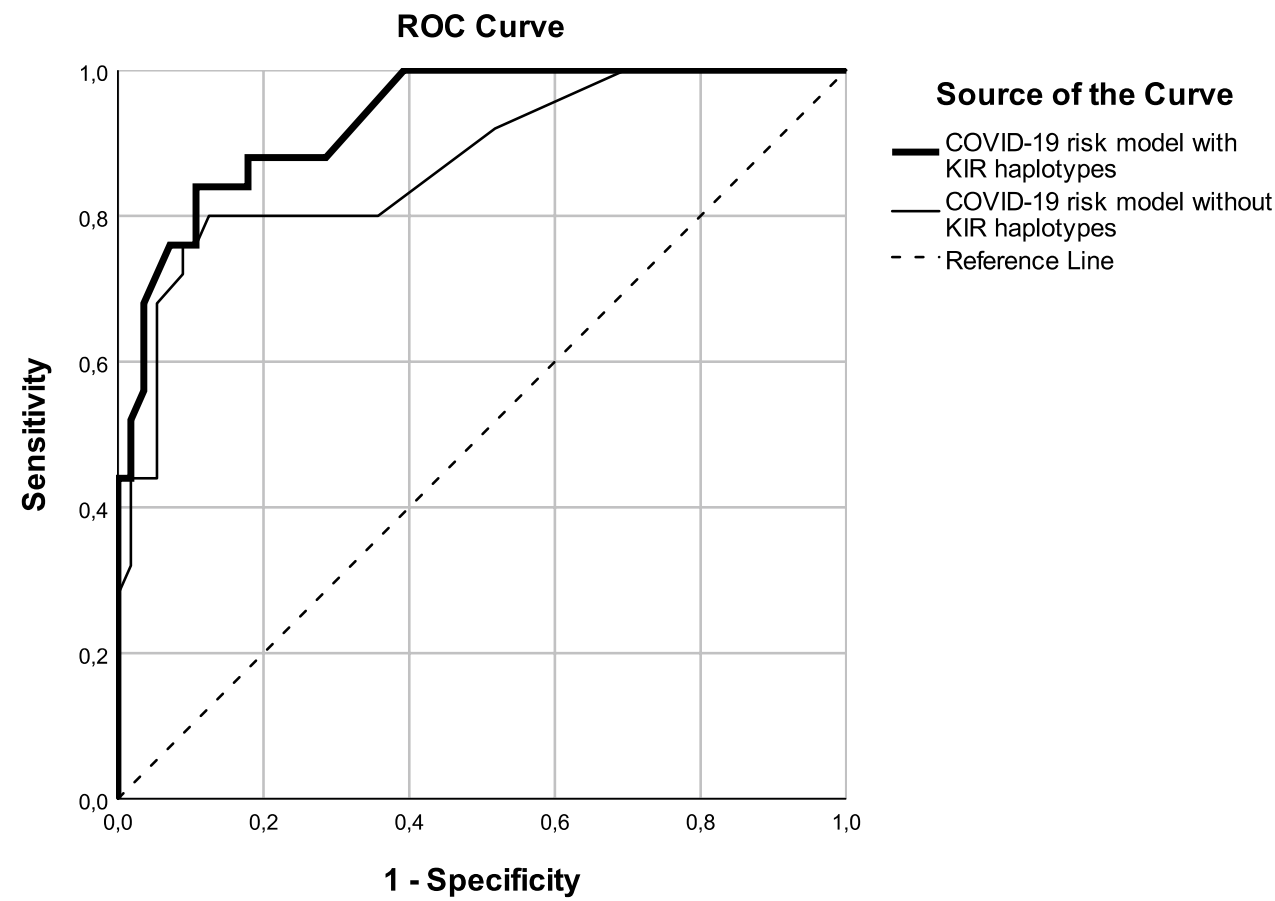


Table 2 Significant independent variables for prediction of severe disease risk (multivariate binary logistic regression). OR: odds ratio

\begin{tabular}{|c|c|c|c|c|c|}
\hline \multirow[t]{2}{*}{ Variables included in the final model } & \multirow[t]{2}{*}{$\mathrm{B}^{\mathrm{A}}$} & \multirow[t]{2}{*}{ Sig } & \multirow[t]{2}{*}{ OR } & \multicolumn{2}{|c|}{ 95\% CI for OR } \\
\hline & & & & Lower & Upper \\
\hline Age $(<35,35-50,51-64, \geq 65)$ & 1.56 & 0.002 & 4.75 & 1.80 & 12.55 \\
\hline Blood type A vs others & -2.74 & 0.01 & 0.07 & 0.01 & 0.52 \\
\hline Number of comorbidities & 1.26 & 0.022 & 3.52 & 1.20 & 10.32 \\
\hline tAA with ligand (Bw4 + ABw4+) & 3.17 & 0.016 & 23.78 & 1.83 & 309.91 \\
\hline $\mathrm{tAB} 1$ with ligand $(\mathrm{C} 2+\mathrm{Bw} 4+)$ & -2.46 & 0.026 & 0.09 & 0.01 & 0.74 \\
\hline Constant & -3.52 & 0.001 & & & \\
\hline
\end{tabular}

${ }^{\mathrm{A}}$ Logistic regression coefficient dyspnea, loss of consciousness, the number of comorbidities, cancer history, neutrophil: lymphocyte ratio (NLR), LDH and direct bilirubin (Liang et al. 2020). The AUC for the correct prediction of the development of critical illness in their study was 0.75 (95\% CI 0.70-0.80), indicating a lower classification performance when compared to our model $(0.93 ; P<0.0001,(95 \%$ CI $0.88,0.99))$. All patients included in their study had already been hospitalized. $\mathrm{Hu}$ et al. (2020) included a total of 40 patients of which 19 were mild and 21 severe cases in a univariate and multivariate analysis which found age, hypertension, lymphopenia, hypoalbuminemia, and elevated NLR to be independent risk factors for severe COVID-19. ROC curves demonstrated age, lymphocyte count, albumin, and NLR to be significant predictive factors for severe COVID-19. The sensitivity and specificity of this novel prediction model for predicting severe COVID-19 were $90.5 \%$ and $84.2 \%$, respectively. In comparison to our model, this model has a higher sensitivity but lower specificity. As conceded by the authors, this study has several limitations including the small sample size and the retrospective approach (Hu et al. 2020). In another similar approach, Ji et al. (Ji et al. 2020) analyzed 208 patients (stable group, $n=168$; progressive group, $n=40$ ). In univariate and multivariate analyses, comorbidities, older age, lower lymphocyte count, and higher LDH were found to be independent risk factors for COVID-19 progression, and a novel scoring model, named as CALL, with an AUC 0.91 (95\% CI 0.86-0.94) was developed. The sensitivity and specificity of the scoring model (with the cutoff of 6) were $95 \%$ and $78 \%$ respectively, suggesting higher sensitivity and similar specificity when compared with our model (Ji et al. 2020).

Table 3 Worldwide distribution of the most common KIR genotypes (Human Genome Diversity Project) ${ }^{\mathrm{A}}$ and comparison with the Turkish population

\begin{tabular}{|c|c|c|c|c|c|c|c|c|c|}
\hline \multicolumn{9}{|c|}{ Human Genome Project Data ${ }^{\mathrm{A}}$} & \multirow[t]{2}{*}{ Turkey } \\
\hline $\begin{array}{l}\text { Centromeric/telomeric } \\
\text { genotype }\end{array}$ & Worldwide & Africa & Mideast & Europe & CSAsia & EAsia & Oceania & America & \\
\hline $\mathrm{cAA} / \mathrm{tAA}$ & $31.40 \%$ & $32.70 \%$ & $37.00 \%$ & $28.40 \%$ & $27.80 \%$ & $46.00 \%$ & $0.00 \%$ & $24.00 \%$ & $23.54 \%$ \\
\hline $\mathrm{cAA} / \mathrm{tAB}$ & $11.00 \%$ & $1.80 \%$ & $10.10 \%$ & $10.10 \%$ & $11.10 \%$ & $16.00 \%$ & $5.40 \%$ & $18.00 \%$ & $11.89 \%$ \\
\hline $\mathrm{cAB} 2 / \mathrm{tAA}$ & $8.50 \%$ & $8.80 \%$ & $18.50 \%$ & $12.20 \%$ & $3.90 \%$ & $8.40 \%$ & $0.00 \%$ & $6.00 \%$ & $13.11 \%$ \\
\hline $\mathrm{cAB} 1 / \mathrm{tAA}$ & $7.20 \%$ & $8.00 \%$ & $21.00 \%$ & $5.40 \%$ & $11.70 \%$ & $2.50 \%$ & $2.70 \%$ & $0.00 \%$ & $10.44 \%$ \\
\hline $\mathrm{cAB} 1 / \mathrm{tAB}$ & $6.40 \%$ & $2.70 \%$ & $9.20 \%$ & $17.60 \%$ & $8.30 \%$ & $1.30 \%$ & $5.40 \%$ & $2.00 \%$ & $17.23 \%$ \\
\hline $\mathrm{cAB} 2 / \mathrm{tAB}$ & $5.0 \%$ & $0.0 \%$ & $3.40 \%$ & $6.80 \%$ & $2.80 \%$ & $3.40 \%$ & $2.70 \%$ & $21.00 \%$ & $7.28 \%$ \\
\hline $\mathrm{tAA}^{\mathrm{B}}$ & $47.10 \%$ & $49.50 \%$ & $76.50 \%$ & $46.00 \%$ & $43.40 \%$ & $56.90 \%$ & $2.70 \%$ & $30.00 \%$ & $50.97 \%$ \\
\hline tAA w ligands & & & & & & & & & $17.73 \%$ \\
\hline $\mathrm{tAB}^{\mathrm{B}}$ & $22.40 \%$ & $4.50 \%$ & $22.70 \%$ & $34.50 \%$ & $22.20 \%$ & $20.70 \%$ & $13.50 \%$ & $41.00 \%$ & $40.53 \%$ \\
\hline $\mathrm{tAB} 1^{\mathrm{C}}$ & & & & & & & & & $29.13 \%$ \\
\hline tAB1 w ligands ${ }^{C}$ & & & & & & & & & $17.48 \%$ \\
\hline $\mathrm{tBB}$ & & & & & & & & & $5.83 \%$ \\
\hline tBB w ligands & & & & & & & & & $4.81 \%$ \\
\hline $\mathrm{cAB} 1^{\mathrm{B}}$ & $13.60 \%$ & $10.70 \%$ & $30.20 \%$ & $23.00 \%$ & $20.00 \%$ & $3.80 \%$ & $8.10 \%$ & $2.00 \%$ & $32.28 \%$ \\
\hline cAB1 w ligands & & & & & & & & & $24.04 \%$ \\
\hline
\end{tabular}

A Adapted from Hollenbach et al. (2012)

${ }^{\mathrm{B}}$ Estimates calculated from worldwide distribution

${ }^{\mathrm{C}}$ In this current study, we have applied an updated version of the telomeric genotype combination (20) 
As confirmed in our study, age and comorbidities are highly predictive of the severity of COVID-19 infection (Schultze and Aschenbrenner 2021; Ko et al. 2020). The role of blood groups has been addressed in many studies though there is, as yet, no consensus (Severe Covid et al. 2020; Hoiland et al. 2020; Latz et al. 2020; Zietz et al. 2020). Two recent studies from Turkey did not reveal any significant associations. Goker et al. (2020) found blood group A to be associated with increased susceptibility to COVID, but not with clinical outcomes. Conversely, Dal et al. (2021) observed a borderline increase in the frequency of blood group $\mathrm{O}$ among pregnant COVID-19 cases. In a study reported in Nature Communications, blood group A was found to be associated with an increased risk of acquiring COVID-19 though appeared to be protective against the need for intubation (Zietz et al. 2020). In our study, we observed a gradual decrease in group A frequency as disease severity increased.

We observed that the risk of severe disease is higher among patients with the tAA motif $(\mathrm{OR}=23.78)$ and was low among patients with $\mathrm{tAB} 1(\mathrm{OR}=0.09)$ when compared with asymptomatic participants (Fig. 1). In our first prediction attempt, we were able to build a model based on age, gender, blood group, and number of comorbidities which reached a specificity of $89.29 \%$ and a sensitivity of 76\% (AUC: $0.87, P<0.0001$ ). After adding tAA genotype and $\mathrm{CAB} 1$ to the model, the specificity increased to $92.86 \%$ and the sensitivity remained the same at $76 \%$ (AUC:0.93, $P<0.0001)$. When compared with the previous clinical models, our model has among the best sensitivity and excellent specificity (Hu et al. 2020; Shan et al. 2018).

According to an earlier report on the worldwide distribution of KIR haplotypes, it is possible to observe populationbased differences; for example, tAA genotypes are more frequent in Asian countries (Maucourant et al. 2020). In our study, ligand-independent tAA frequency in Turkey (28.6\%) is observed at a similar frequency as in the Central-South Asian populations (Table 3). Occurrence with its ligands was seen less frequently (17.7\%) in our cohort. On the other hand, our donor $\mathrm{ABB} 1$ frequency shares similarities with both European and Central-South Asian populations (Table 3). However, similar data on KIR-ligand combos does not exist to allow comparison (17.48\%). Likewise, blood group frequencies vary across populations (Dal et al. 2021). The incidence of severe COVID-19 is generally accepted to be less than $15 \%$ and is influenced by well-known risk factors such as age and comorbidities. In the final update of COVID-19 Pandemic Planning Scenarios, CDC presented the frequencies of asymptomatic cases, ranging from 15 to 30\% (Oran and Topol 2020; Oran and Topol 2021; CDC 2019). The model constructed in our study included both age, comorbidities, and blood group as well as the telomeric genotypes tAA and $\mathrm{tAB} 1$ but not gender. The increase in the frequency of tAA in severe cases and the decrease in $\mathrm{tAB} 1$ in asymptomatic patients are insufficient to allow for the prediction of the severity of COVID-19 severity. However, when combined with demographics, our prediction model had a sensitivity of $76 \%$ and a specificity of $92.86 \%$.

As detailed in the introduction, none of the virus-KIR association studies have integrated telomeric/centromeric KIR genotypes into their analyses. However, their results suggest that $\mathrm{tAB} 1$ with or without its ligand is associated with less severe disease during DENV, HIV, and H1N1 infection (Ahlenstiel et al. 2008; La et al. 2011). Furthermore, HIV, $\mathrm{HCV}$, and CHIKV infection-KIR association results are also suggestive of a protective effect of the cAA motif (Mori et al. 2019; Ahlenstiel et al. 2008; Jiang et al. 2013). In our study, $\mathrm{tAB} 1$ and $\mathrm{cAA}$ were found to be protective; however, in the model, only tAB1 was significant. The number of activating KIR genes in the $\mathrm{tAB} 1$ as opposed to the cAA motif may have contributed to increased protection against all these mostly RNA viruses. None of the earlier similar studies reported any associations with the tAA genotype, an inhibitory KIR gene dominant motif. Conversely, one may deduce that the tBB genotype behaves as a protective variant based on our findings. In our study, the frequency of tBB is too low to allow a significant effect in the prediction model. However, KIR2DS5, an activating KIR motif that has surface expression and is included in the B genotype, was less frequently seen in severe cases when compared to asymptomatic ones.

In conclusion, the integration of KIR genotypes with previously recognized risk variables in a novel risk prediction model was able to classify COVID-19 infection risk successfully. We do not yet know the role of Neanderthal immune response-related genes in this model. A weakness of our study is its size and limited geographical location. Nevertheless, a model that does not include acute phase reactants has the distinct advantage of allowing for the recognition of individuals before the onset of infection. Nonetheless, further validation and refinement of the risk models are clearly required.

\section{Methods}

\section{Participants}

After Ethical Committee approval (Ministry of Health, 29.04.2020; Local Ethical Committee, 14.05.2020) and with a planned study design, 52 asymptomatic, 27 mildintermediate, and 25 additional severely ill hospitalized patients diagnosed with COVID-19 infection confirmed with PCR tests were consented prior to inclusion in the study. Assignment of patients according to asymptomatic, mild-intermediate, or severe was determined according to the WHO (2020) guidelines. During the first surge of infection between August and December 2020, blood samples were collected for genotyping of KIR and KIR-ligands using Olerup KIR Genotyping and KIR/HLA Ligand typing kits 
respectively (Olerup, Stockholm, Sweden; Cat No: 104.101$12 \mathrm{u}$ and 104.201-12u). KIR haplotype motifs were determined according to the combinations presented by Cisneros et al. (2020). KIR gene variations derived from Human Genome Project data were used as a comparison of frequencies across populations worldwide (Hollenbach et al. 2012).

Age, gender, comorbidities, blood groups, and clinical and biochemical parameters were recorded. KIR and HLAtyped donors registered since 2010 in Ankara University Donor Registry ( $n$ : 449) with a median age of 43 (25-76) were searched in the COVID-19 database (under the auspices of the Ministry of Health of Turkey) or called by phone to determine if they had been diagnosed with COVID-19 during the study duration. Thirty-seven had COVID-19 history, of which 28 could be retrieved for clinical severity: four asymptomatic and 24 with mild-intermediate symptoms not requiring hospitalization. Mortality was detected only among the first cohort ( $n=8$ patients). Thus, the first and second cohorts were combined to reach 132 patients in this association study (Table 1). Asymptomatic patients were diagnosed mostly during screening for COVID-19 exposed contacts.

\section{Statistics}

The distribution of KIR genotypes (w/wo ligands), blood groups, gender, and comorbidity frequencies among the asymptomatic, mild-intermediate, and severe cases was evaluated using chi-square or two-tailed Fisher's exact tests as appropriate. For continuous variables (age, D-dimer, C-reactive protein), either one-way ANOVA or the Kruskal-Wallis test was used according to the distribution of the variables. In the selection of the candidate variables for multivariate analysis, the significance level was set at 0.10 in univariate tests. Age, blood type $\mathrm{A}$, number of comorbidities, $\mathrm{tAB} 1$ with ligands $\mathrm{C} 2+\mathrm{Bw} 4$, tAA with ligands $\mathrm{Bw} 4+\mathrm{ABw} 4$, and $\mathrm{CAB} 1$ with ligands $\mathrm{C} 1+\mathrm{C} 2$ were selected as candidate variables, and a multivariate binary logistic regression model was built and performed by backward elimination method. The area under the ROC curve (AUC) was used to evaluate the discriminative ability of the prediction model between severe and asymptomatic cases. All statistical analyses were performed using IBM SPSS Statistics (version 26; IBM Corporation, Armonk, NY, USA).

\section{Study approval}

In Turkey, all COVID-19 clinical research studies are submitted for approval from the Ministry of Health prior to the Institutional Ethical Committee submission. This study has been approved by the Ministry of Health (29.04.2020) and Ankara University Ethical Committee (14.05.2020; İ5-266-20).
Acknowledgements The authors would like to acknowledge the research grants received from Ankara University and Turkish Academy of Sciences.

Author contribution $\mathrm{MB}$, OM, and GC designed the study, EBGO and HYA prepared the study proposal, and IAK, EG, GC, and GCS participated in the diagnosis of COVID-19 patient clinical data recruitment. MY contributed to data analysis and EK and HYA performed the statistical analysis and the risk modelling. MB is the director of Ankara University Donor Registry. KD is the director of the Immunogenetics Laboratory; she is also the immune-geneticist responsible for the KIR and KIR ligand molecular analysis and interpretation of results plus genotype assignments; MB wrote the manuscript. All authors participated in the revision of the manuscript. EK and KD are experts in their fields and have spent equal amount of time and effort during the study result recruitment and analysis. Both have contributed equally preventing a fair order assignment.

Funding The study is funded by Ankara University Scientific Research Grants (no: 20Y0901012) and the Turkish Academy of Sciences.

\section{Declarations}

Conflict of interest The authors declare no competing interests.

\section{References}

Ahlenstiel G, Martin MP, Gao X, Carrington M, Rehermann B (2008) Distinct KIR/HLA compound genotypes affect the kinetics of human antiviral natural killer cell responses. J Clin Invest 118(3):1017-1026

Aranda-Romo S, Garcia-Sepulveda CA, Comas-Garcia A, Lovato-Salas F, Salgado-Bustamante M, Gomez-Gomez A et al (2012) Killercell immunoglobulin-like receptors (KIR) in severe A (H1N1) 2009 influenza infections. Immunogenetics 64(9):653-662

Beksac K, Beksac M, Dalva K, Karaagaoglu E, Tirnaksiz MB (2015) Impact of "Killer Immunoglobulin-Like Receptor/Ligand" genotypes on outcome following surgery among patients with colorectal cancer: activating KIRs are associated with long-term disease free survival. PLoS One 10(7):e0132526

Casanova JL, Su HC, Effort CHG (2020) A global effort to define the human genetics of protective immunity to SARS-CoV-2 infection. Cell 181(6):1194-1199

CDC (2021) COVID-19 Pandemic Planning Scenarios. https://www. cdc.gov/coronavirus/2019-ncov/hcp/planning-scenarios.html. Updated 19.03.2021 Accessed 02.05.2021

Chaisri S, Jumnainsong A, Romphruk A, Leelayuwat C (2020) The effect of KIR and HLA polymorphisms on dengue infection and disease severity in northeastern Thais. Med Microbiol Immunol 209(5):613-620

Cisneros E, Moraru M, Gomez-Lozano N, Muntasell A, Lopez-Botet M, Vilches C (2020) Haplotype-based analysis of KIR-gene profiles in a South European population-distribution of standard and variant haplotypes, and identification of novel recombinant structures. Front Immunol 11:440

Dal MS, Ata N, Altuntaş F, Başci S, Yiğgenoğlu TN, Korkmaz S et al (2021) COVID-19 Clinical course and blood groups: Turkish population-based study. Turk J Med Sci

Geng MJ, Wang LP, Ren X, Yu JX, Chang ZR, Zheng CJ et al (2021) Risk factors for developing severe COVID-19 in China: an analysis of disease surveillance data. Infect Dis Poverty 10(1):48

Goker H, Aladag Karakulak E, Demiroglu H, Ayaz Ceylan CM, Buyukasik Y, Inkaya AC et al (2020) The effects of blood group 
types on the risk of COVID-19 infection and its clinical outcome. Turk J Med Sci 50(4):679-683

Hoiland RL, Fergusson NA, Mitra AR, Griesdale DEG, Devine DV, Stukas S et al (2020) The association of ABO blood group with indices of disease severity and multiorgan dysfunction in COVID19. Blood Adv 4(20):4981-4989

Hollenbach JA, Nocedal I, Ladner MB, Single RM, Trachtenberg EA (2012) Killer cell immunoglobulin-like receptor (KIR) gene content variation in the HGDP-CEPH populations. Immunogenetics 64(10):719-737

Hu H, Du H, Li J, Wang Y, Wu X, Wang C et al (2020) Early prediction and identification for severe patients during the pandemic of COVID-19: a severe COVID-19 risk model constructed by multivariate logistic regression analysis. J Glob Health10(2):020510

Jennes W, Verheyden S, Demanet C, Adje-Toure CA, Vuylsteke B, Nkengasong JN et al (2006) Cutting edge: resistance to HIV-1 infection among African female sex workers is associated with inhibitory KIR in the absence of their HLA ligands. J Immunol 177(10):6588-6592

Ji D, Zhang D, Xu J, Chen Z, Yang T, Zhao P et al (2020) Prediction for progression risk in patients with COVID-19 pneumonia: the CALL score. Clin Infect Dis 71(6):1393-1399

Jiang Y, Chen O, Cui C, Zhao B, Han X, Zhang Z et al (2013) KIR3DS1/ L1 and HLA-Bw4-80I are associated with HIV disease progression among HIV typical progressors and long-term nonprogressors. BMC Infect Dis 13:405

Khakoo SI, Carrington M (2006) KIR and disease: a model system or system of models? Immunol Rev 214:186-201

Khakoo SI, Thio CL, Martin MP, Brooks CR, Gao X, Astemborski J et al (2004) HLA and NK cell inhibitory receptor genes in resolving hepatitis $C$ virus infection. Science 305(5685):872-874

Khodeir MM, Shabana HA, Alkhamiss AS, Rasheed Z, Alsoghair M, Alsagaby SA et al (2021) Early prediction keys for COVID19 cases progression: a meta-analysis. J Infect Public Health 14(5):561-569

Ko JY, Danielson ML, Town M, Derado G, Greenlund KJ, Daily Kirley P et al (2020) Risk factors for COVID-19-associated hospitalization: COVID-19-associated hospitalization surveillance network and behavioral risk factor surveillance system. Clin Infect Dis

La D, Czarnecki C, El-Gabalawy H, Kumar A, Meyers AF, Bastien $\mathrm{N}$ et al (2011) Enrichment of variations in KIR3DL1/S1 and KIR2DL2/L3 among H1N1/09 ICU patients: an exploratory study. PLoS One 6(12):e29200

Latz CA, DeCarlo C, Boitano L, Png CYM, Patell R, Conrad MF et al (2020) Blood type and outcomes in patients with COVID-19. Ann Hematol 99(9):2113-2118

Liang W, Liang H, Ou L, Chen B, Chen A, Li C et al (2020) Development and validation of a clinical risk score to predict the occurrence of critical illness in hospitalized patients with COVID-19. JAMA Intern Med 180(8):1081-1089

Lu Z, Zhang B, Chen S, Gai Z, Feng Z, Liu X et al (2008) Association of KIR genotypes and haplotypes with susceptibility to chronic hepatitis B virus infection in Chinese Han population. Cell Mol Immunol 5(6):457-463

Martin MP, Gao X, Lee JH, Nelson GW, Detels R, Goedert JJ et al (2002) Epistatic interaction between KIR3DS1 and HLA-B delays the progression to AIDS. Nat Genet 31(4):429-434

Maucourant C, Filipovic I, Ponzetta A, Aleman S, Cornillet M, Hertwig L et al (2020) Natural killer cell immunotypes related to COVID-19 disease severity. Science Immunology 5(50):eabd6832

Mori M, Leitman E, Walker B, Ndung'u T, Carrington M, Goulder P (2019) Impact of HLA allele-KIR pairs on HIV clinical outcome in South Africa. J Infect Dis 219(9):1456-1463

Oran DP, Topol EJ (2020) Prevalence of asymptomatic SARS-CoV-2 infection : a narrative review. Ann Intern Med 173(5):362-367
Oran DP, Topol EJ (2021) The proportion of SARS-CoV-2 infections that are asymptomatic : a systematic review. Ann Intern Med

Ovsyannikova IG, Haralambieva IH, Crooke SN, Poland GA, Kennedy RB (2020) The role of host genetics in the immune response to SARS-CoV-2 and COVID-19 susceptibility and severity. Immunol Rev 296(1):205-219

Pairo-Castineira E, Clohisey S, Klaric L, Bretherick AD, Rawlik K, Pasko D et al (2021) Genetic mechanisms of critical illness in COVID-19. Nature 591(7848):92-98

Petitdemange C, Wauquier N, Jacquet JM, Theodorou I, Leroy E, Vieillard V (2014) Association of HLA class-I and inhibitory KIR genotypes in Gabonese patients infected by Chikungunya or Dengue type-2 viruses. PLoS One 9(9):e108798

Rod JE, Oviedo-Trespalacios O, Cortes-Ramirez J (2020) A briefreview of the risk factors for COVID-19 severity. Rev Saude Publica 54:60

Roe D, Vierra-Green C, Pyo CW, Eng K, Hall R, Kuang R et al (2017) Revealing complete complex KIR haplotypes phased by long-read sequencing technology. Genes Immun 18(3):127-134

Sahin U, Dalva K, Gungor F, Ustun C, Beksac M (2018) Donor-recipient killer immunoglobulin like receptor (KIR) genotype matching has a protective effect on chronic graft versus host disease and relapse incidence following HLA-identical sibling hematopoietic stem cell transplantation. Ann Hematol 97(6):1027-1039

Schultze JL, Aschenbrenner AC (2021) COVID-19 and the human innate immune system. Cell 184(7):1671-1692

Severe Covid-19 GWAS Group, Ellinghaus D, Degenhardt F, Bujanda L, Buti M, Albillos A et al (2020) Genomewide association study of severe COVID-19 with respiratory failure. N Engl J Med 383(16):1522-1534

Shan Z, Huang J, Liao Q, Huang K, Wang M, Xu R et al (2018) Association of killer cell immunoglobulin-like receptors with spontaneous clearance of hepatitis $\mathrm{C}$ virus in the Chinese population. Transfusion 58(4):1028-1035

Soleimanian S, Yaghobi R (2020) Harnessing memory NK cell to protect against COVID-19. Front Pharmacol 11:1309

The COVID-19 Host Genetics Initiative (2020) The COVID-19 Host Genetics Initiative, a global initiative to elucidate the role of host genetic factors in susceptibility and severity of the SARS-CoV-2 virus pandemic. Eur J Hum Genet. 28(6):715-8

van der Made CI, Simons A, Schuurs-Hoeijmakers J, van den Heuvel G, Mantere T, Kersten S et al (2020) Presence of genetic variants among young men with severe COVID-19. JAMA

Vendelbosch S, de Boer M, van Leeuwen K, Pourfarzad F, Geissler J, van den Berg TK et al (2015) Novel insights in the genomic organization and hotspots of recombination in the human KIR locus through analysis of intergenic regions. Genes Immun 16(2):103-111

Vetter P, Vu DL, L'Huillier AG, Schibler M, Kaiser L, Jacquerioz F (2020) Clinical features of COVID-19. BMJ369:m1470

WHO (2020) Novel Coronavirus. Situation Report 205:1-19

Wu SC, Arthur CM, Wang J, Verkerke H, Josephson CD, Kalman D et al (2021) The SARS-CoV-2 receptor-binding domain preferentially recognizes blood group A. Blood Adv 5(5):1305-1309

Zeberg HPS (2020) Risk Variant for severe COVID-19 inherited from Neanderthals. Am J Med Genet A 182(10):2203-2204

Zeberg H, Paabo S (2020) The major genetic risk factor for severe COVID-19 is inherited from Neanderthals. Nature 587(7835):610-612

Zeberg H, Paabo S (2021) A genomic region associated with protection against severe COVID-19 is inherited from Neandertals. Proc Natl Acad Sci U S A. 118(9)

Zhou F, Yu T, Du R, Fan G, Liu Y, Liu Z et al (2020) Clinical course and risk factors for mortality of adult inpatients with COVID19 in Wuhan, China: a retrospective cohort study. Lancet 395(10229):1054-1062 
Zhou S, Butler-Laporte G, Nakanishi T, Morrison DR, Afilalo J, Afilalo $M$ et al (2021) A Neanderthal OAS1 isoform protects individuals of European ancestry against COVID-19 susceptibility and severity. Nat Med 27(4):659-667

Zietz M, Zucker J, Tatonetti NP (2020) Associations between blood type and COVID-19 infection, intubation, and death. Nat Commun 11(1):5761
Publisher's Note Springer Nature remains neutral with regard to jurisdictional claims in published maps and institutional affiliations. 